\title{
Studies of Magnetic Structure of Cobalt-ferrite Nano-particles
}

\author{
T. M. Meaz, M. A. Amer and M. K. El-Nimr
}

Physics Department, Faculty of Science, Tanta University, Tanta, Egypt.

By means of Mössbauer effect spectroscopy, ultrafine cobalt-ferrite particles was studied. The spectra were obtained between $14 \mathrm{~K}$ and $300 \mathrm{~K}$, and also at $5 \mathrm{~K}$ with $4 \mathrm{~T}$ applied external magnetic field. The spectra obtained with large magnetic fields demonstrated the non-collinear magnetic structure occurs in the surface layers of the Co-ferrite system. The importance of the applied field during the measurements for a correct characterization and the coating effect were shown. The measurements illustrated that coating the surface of Co$\mathrm{Fe}_{2} \mathrm{O}_{4}$ particles with oleic acid does not necessarily explain the canting of spins observed.

\section{Introduction:}

It is known that nanoscale materials show properties that are often significantly different from the bulk. The magnetic properties of fine particles are influenced by various effects such as single domain structure, superparamagnetism, and surface effects [1-3].

Cobalt ferrite has the spinel structure of magnetite, $\mathrm{Fe}_{3} \mathrm{O}_{4}$, with $\mathrm{Fe}^{3+}$ ions distributed among tetrahedral A and octahedral B sites. Oxidation of magnetite produces vacancies, which may be on either the A or B sites although B site is more common [4]. For cobalt substitution it was found that $\mathrm{B}$ site is preferentially occupied by Co ions [5]. Cobalt ferrite is ferrimagnetic and of industrial importance for recording tapes. Mössbauer effect and magnetization measurements of cobalt ferrite particles have shown that the magnetic properties depend on how they were prepared [5-10]. A decrease in a saturation magnetization was observed by decreasing the particle size [7]. Spin canting effect was observed for coated Co-ferrite particles [7]. It has been shown that the magnetization could be reversibly reduced, and the non-collinearity could be increased by adsorbing surfactant molecules onto the particle surface. Many invistigatoure have been studied cobalet ferrite systems [11-19]. 
In this investigation nano- particles of $\mathrm{CoFe}_{2} \mathrm{O}_{4}$ with average diameters of 10.3 and $4 \mathrm{~nm}$ were studied. The role of the applied magnetic field during Mössbauer measurments of the cobalt ferrite system was discussed. The results can be explained with the assumption of a surface spin canted layer. The influence of coating is also discussed.

\section{Experimental:}

\subsection{Sample Preparations:}

1) Uncoated samples CO2: Magnetic particles of cobalt ferrite were prepared by controlled addition with stirring [11] of an aqueous mixture of $100 \mathrm{ml}$ of $1 \mathrm{M}$ cobalt sulfate and $100 \mathrm{ml}$ of $2 \mathrm{M} \mathrm{Fe}(\mathrm{III})$ nitrate to an excess $(950 \mathrm{ml})$ of $1 \mathrm{M} \mathrm{KOH}$ solution at $0^{\circ} \mathrm{C}$. Then the mixture was heated for 20 minutes at $95^{\circ} \mathrm{C}$.

2) Coated samples CO3: For the coated samples, on cooling, oleic acid was added and then the whole system stirred for 1 hour.

3) Coated small particles samples CO1: In this sample, the oleic acid was added prior to heating.

\subsection{Measuring Techniques:}

Structural analysis of the samples was performed by means of X-ray diffraction, electron microscopy and Mössbauer effect spectroscopy. X-ray diffraction measurements were carried out using a Philips PW1130/90 X-ray diffractometer $\left(\mathrm{CuK}_{\alpha}\right.$ radiation with a graphite monochromator mounted in the diffracted beam). The morphology and particle size have been previously investigated using a Philips EM-301 TEM [20].

Mössbauer spectra were obtained by means of a conventional constant acceleration Mössbauer spectrometer with a $50 \mathrm{mCi}$ source of ${ }^{57} \mathrm{Co}$ in $\mathrm{Rh}$. The spectrometer was calibrated using a $12.5 \mu \mathrm{m}$ foil of $\alpha$-Fe at room temperature. The spectra were obtained at different temperatures with and without an applied magnetic field parallel or perpendicular to the $\gamma$-ray direction.

\section{Results:}

\subsection{Structure Analysis:}

The X-ray diffractograph exhibits line broadening associated with a fine particle system. A fit of the experimental data yields a value of the lattice constant of $8.393 \pm 0.005 \AA$ consistent with bulk cobalt ferrite $(8.39 \pm 0.005 \AA$ [21]). There is no evidence of any other impurity phases present. The particle sizes were obtained by X-ray diffractographs using the Debye-Scherrer expression and by electron micrograph. The average diameter of the particles was estimated to be $10.3 \mathrm{~nm}$ for $\mathrm{CO} 2$ and $\mathrm{CO} 3$ samples, and $4 \mathrm{~nm}$ for $\mathrm{CO} 1$ sample. 


\subsection{Mössbauer Results:}

Spectra of the cobalt-ferrite samples were obtained at different temperatures (Figs. (1)). The spectra obtained at room temperature for the three samples show a magnetic splitting sextet in addition to a quadrupole doublet. The relative area of the doublet for the coated samples CO3 was slightly larger than that for the uncoated samples $\mathrm{CO} 2$, while doublet area was much larger for the sample CO1. The average hyperfine fields at $295 \mathrm{~K}$ were found to be 45.4 $\mathrm{T}, 45.3 \mathrm{~T}$ and $45.2 \mathrm{~T}$ for the sample $\mathrm{CO} 1, \mathrm{CO} 2$ and $\mathrm{CO} 3$, respectively. The isomer shift is $0.32 \mathrm{mms}^{-1}$ and the quadrupole shifts are only magnetically split components were observed in the two samples $\mathrm{CO} 2$ and $\mathrm{CO} 3$, while for sample $\mathrm{CO} 1$ a sextet and a quadrupole doublet are observed. This was not observed at $14 \mathrm{~K}$ while the magnetically split component is observed.
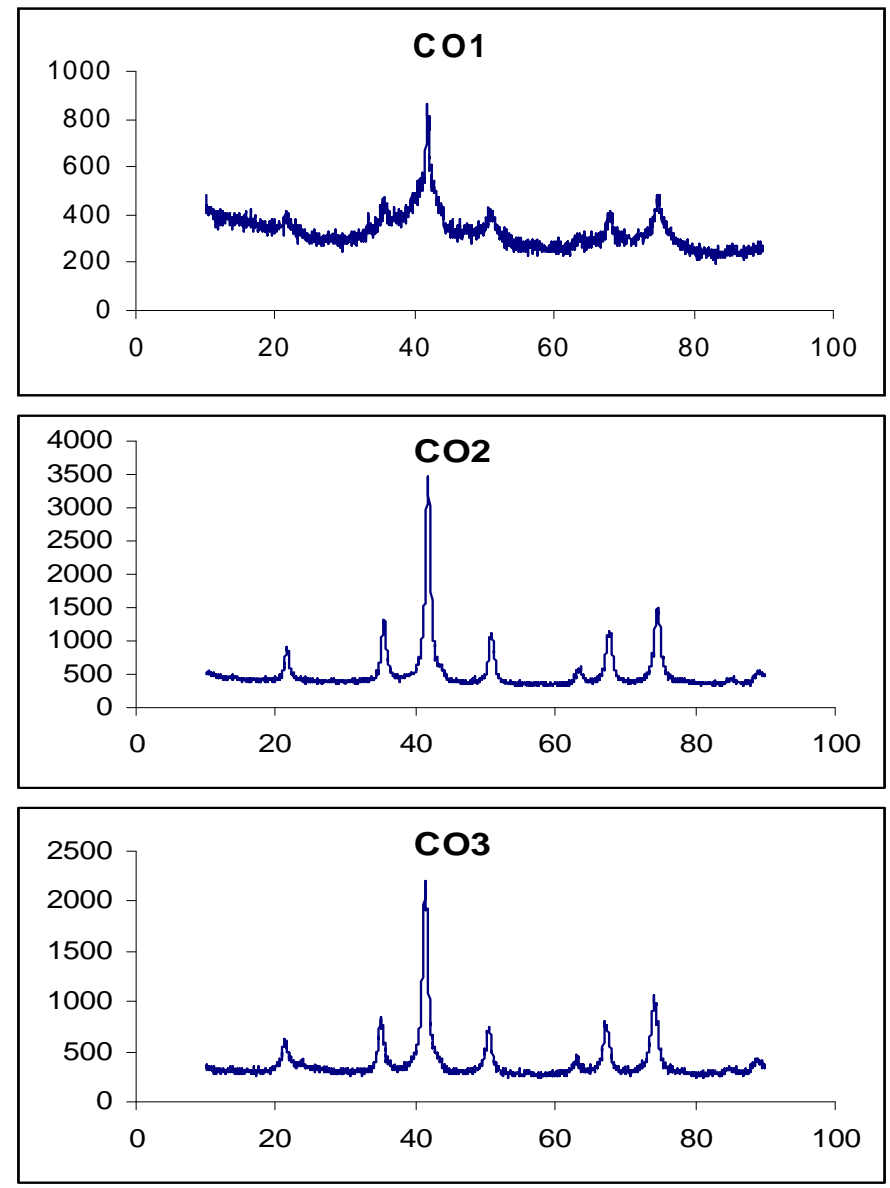

Fig. (1): X-ray diffraction patterns obtained at room temperature for indicated samples. 
Mössbauer spectra were also obtained at $80 \mathrm{~K}$ with an applied magnetic field perpendicular to the $\gamma$-ray direction. For all samples an increase in the intensity of lines 2 and 5 were observed with an applied $0.6 \mathrm{~T}$ magnetic field. This observation indicates that magnetic moments of the samples were at least partly aligned with the applied field. The average hyperfine fields at $80 \mathrm{~K}$ are $51.5 \mathrm{~T}$ for the coated samples $\mathrm{CO} 3$ and $51.5 \mathrm{~T}$ for uncoated ones $\mathrm{CO} 2$. At $18 \mathrm{~K}$ these values are $52.2 \mathrm{~T}$ and $52.0 \mathrm{~T}$, respectively. The isomer shift is $0.43 \mathrm{mms}^{-1}$ at $18 \mathrm{~K}$ for both samples. The $18 \mathrm{~K}$ spectra and the $14 \mathrm{~K}$ spectrum show an asymmetry of line intensities similar to that of pure maghemite.

The line width of line 6 , in the sextet increases as the temperature was lowered. This can be understood if at lower temperature the separation between A and B site spectra becomes larger. Similar results have been observed for Alsubstituted maghemite [22] and cobalt ferrite [8,9].

The spectra at $18 \mathrm{~K}$ were also fitted with two sextets with Lorentzian of the spectra, the parameters are listed in Table 1.

Table (1): Isomer shift $\delta$, quadrupole shift $\varepsilon$, hyperfine field $\mathrm{B}_{\mathrm{hf}}$, line width $\Gamma$ (of line 1) and area $\mathrm{A}$ of the indicated samples measured at $18 \mathrm{~K}$.

\begin{tabular}{|l|l|l|l|l|l|l|}
\hline Sample & Site & $\delta\left(\mathrm{mms}^{-1}\right)$ & $\varepsilon\left(\mathrm{mms}^{-1}\right)$ & $\mathrm{B}_{\mathrm{hf}}($ tesla $)$ & $\Gamma\left(\mathrm{mms}^{-1}\right)$ & $\mathrm{A}(\%)$ \\
\hline Uncoated & A & 0.41 & -0.01 & 50.8 & 0.52 & 53.1 \\
CO2 & $\mathrm{B}$ & 0.49 & 0.03 & 53.2 & 0.62 & 46.9 \\
\hline Coated & A & 0.41 & 0.01 & 51.2 & 0.60 & 53.5 \\
CO3 & B & 0.49 & -0.02 & 53.5 & 0.55 & 46.5 \\
\hline
\end{tabular}

The uncertainties for the isomer shift, the quadrupole shift and the line width are $\pm 0.02 \mathrm{mms}^{-1}$, the uncertainty for the hyperfine field is $\pm 0.5 \mathrm{~T}$ and for the area it is $\pm 2 \%$.

Table (2): Isomer shift $\delta$, quadrupole shift $\varepsilon$, hyperfine field $\mathrm{B}_{\mathrm{hf}}$, line width $\Gamma$ (of line 1) and area $\mathrm{A}$ of the indicated samples measured at $5 \mathrm{~K}$ with 4.0 T magnetic field.

\begin{tabular}{|l|l|l|l|l|l|l|}
\hline Sample & Site & $\delta\left(\mathrm{mms}^{-1}\right)$ & $\varepsilon\left(\mathrm{mms}^{-1}\right)$ & $\mathrm{B}_{\mathrm{hf}}$ (tesla) & $\Gamma\left(\mathrm{mms}^{-1}\right)$ & $\mathrm{A}(\%)$ \\
\hline CO3 & A & 0.38 & -0.02 & 54.0 & 0.53 & 28.3 \\
& B & 0.46 & -0.01 & 48.3 & 0.56 & 28.0 \\
& C & 0.48 & 0.02 & 50.2 & 0.51 & 43.7 \\
\hline CO2 & A & 0.36 & -0.02 & 53.9 & 0.53 & 27.5 \\
& B & 0.45 & 0.05 & 48.1 & 0.59 & 26.5 \\
& C & 0.47 & 0.01 & 50.2 & 0.57 & 46.4 \\
\hline CO1 & A & 0.38 & 0.03 & 52.9 & 0.57 & 9.7 \\
& B & 0.48 & 0.00 & 46.8 & 0.56 & 8.2 \\
& C & 0.46 & 0.00 & 49.6 & 1.03 & 82.1 \\
\hline
\end{tabular}

The uncertainties for the isomer shift, the quadrupole shift and the line width are $\pm 0.02 \mathrm{mms}^{-1}$, the uncertainty for the hyperfine field is $\pm 0.5 \mathrm{~T}$ and for the area it is $\pm 2 \%$. 
Figure (2) shows the in-field Mössbauer spectra obtained at $5 \mathrm{~K}$ with 4.0 $\mathrm{T}$ applied magnetic field parallel to the gamma ray direction. An important observation notes in the lines 2 and 5 which does not vanish. The A and B sites separation is now clear in the spectra of samples $\mathrm{CO} 2$ and $\mathrm{CO} 1$, but a considerable overlap remains. For sample CO1 the applied magnetic field only gives rise to some line broadening. The presence of lines 2 and 5 can be attributed to a canting of the spins with respect to the applied magnetic field. The spectra were fitted with three sextets. Two of these sextets correspond to the A and B sites ferrimagnetic phase (in which the spins are aligned parallel and antiparallel to the direction of the applied magnetic field). The third component $\mathrm{C}$ is assigned to the canted spins (in which the spins are noncollinear with the magnetic field). The area of the transition lines with $\Delta \mathrm{m}=0$ were constrained to be zero for the A and B components, while for the canted component $\mathrm{C}$ it was constrained to be $2 / 3$ the area of the related outer lines.
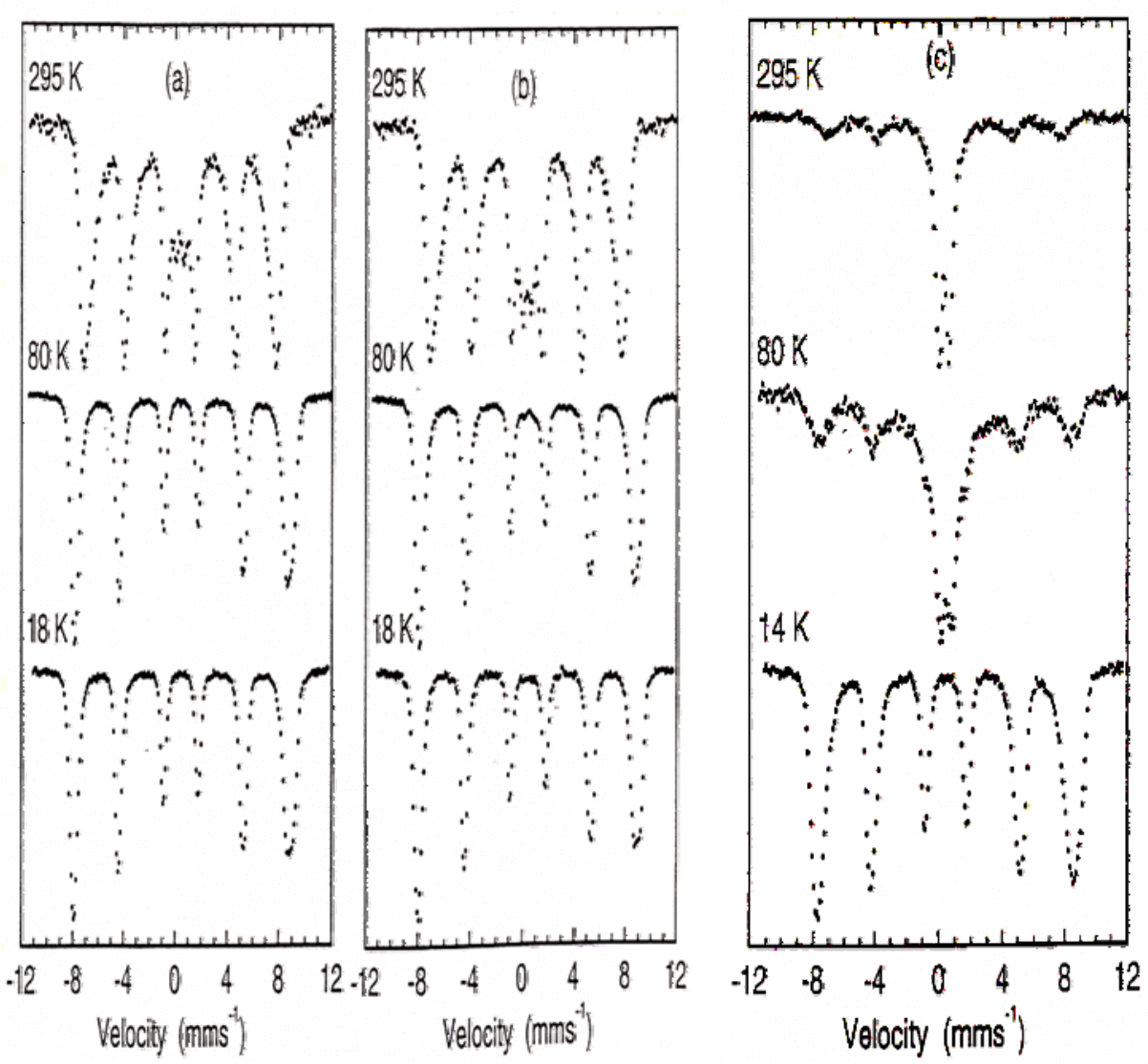

Fig. (2): Mössbauer effect spectra obtained at the indicated temperatures a) uncoated sample $\mathrm{CO} 2, \mathrm{~b}$ ) coated sample $\mathrm{CO} 3$ and c) coated small particle sample $\mathrm{CO} 1$. 
The area ratio of line pairs $(2,5)$ to $(1,6)$ in the in-field spectra at $5 \mathrm{~K}$ for the sum of the subspectra, were estimated to be $1.51: 3,0.73: 3$ and $0.67: 3$ for the samples $\mathrm{CO} 1, \mathrm{CO} 2$ and $\mathrm{CO} 3$, respectively. The hyperfine parameters of the infield spectra are listed in the Table (2).

\section{Discussion:}

At $18 \mathrm{~K}$ the hyperfine fields were found to be $53.2 \mathrm{~T}$ and $50.8 \mathrm{~T}$ for the uncoated samples $\mathrm{CO} 2$, while for the coated sample $\mathrm{CO} 3$ they were $53.4 \mathrm{~T}$ and 51.1, for A and B sites, respectively. The spectra show symmetry of the line intensities similar to that of pure maghemite. Moreover, in these studies the quadrupole shifts are approximately zero.

The application of a $4 \mathrm{~T}$ external magnetic field parallel to the gamma ray direction caused a splitting in the outer lines, while lines 2 and 5 do not vanish. The A and B sites separation is now clear in samples $\mathrm{CO} 3$ and $\mathrm{CO} 2$, but a considerable overlap remains. The influence of the magnetic field is similar to that obtained in earlier studies of cobalt ferrite [7].

The application of an external magnetic field, results in an increase in the A-site hyperfine field and decrease in the B-site hyperfine field. The observed difference in the hyperfine field between A- and B-sites, is 5.7 T. The observed difference could be understood by the following : First, the difference in the hyperfine field between A and B site spectra at $18 \mathrm{~K}$ is $\sim 2.3 \mathrm{~T}$. assuming that the hyperfine fields are saturated at $18 \mathrm{~K}$, then the difference in the hyperfine fields will be the same as $5 \mathrm{~K}$. Second, the B site has larger hyperfine field than that of A site. For example, the hyperfine fields at $18 \mathrm{~K}$ for A and B sites (for example for $\mathrm{CO} 2$ ) are 50.8 and $53.2 \mathrm{~T}$, respectively. At $5 \mathrm{~K}$ in applied magnetic field these values are 53.9 and $48.1 \mathrm{~T}$ for $\mathrm{A}$ and $\mathrm{B}$ sites, respectively. The differences observed due to apply a $4 \mathrm{~T}$ external magnetic field are $3.1 \mathrm{~T}$ and $5.1 \mathrm{~T}$ for the $\mathrm{A}$ and $\mathrm{B}$ sites. These values are, within experimental error, equal to the value of the applied field.

In Fig. (3), the presence of 2 and 5 lines indicate that the spin structure is non-collinear with the direction of the applied field. In the case of ultrafine particles [1], which have a significant proportion of atoms in the surface layers, there is a possibility of magnetic surface reconstruction, whereby the spin of the system lower its energy by adopting a non-collinear structure in the surface layers. An increase in the anisotropy constant as the particle size decreases may be also account. It has been shown that cobalt ferrite has anisotropy constant larger than that of pure maghemite [9]. On the other hand coating the surface with a surfactant may be also contributing [7]. 


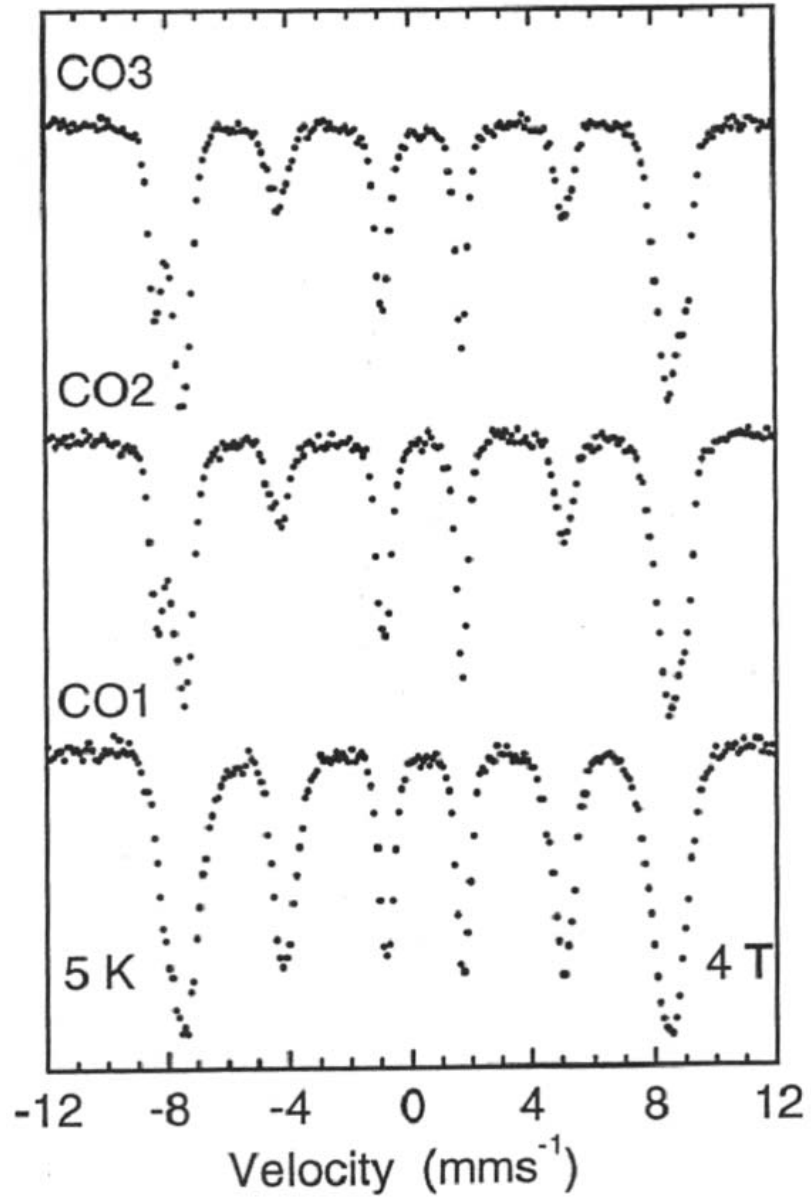

Fig. (3): Mössbauer effect spectra, obtained at $5 \mathrm{~K}$ with $4.0 \mathrm{~T}$ magnetic field applied parallel to the $\gamma$-ray direction for the indicated samples.

All these parameters could contribute to the spin canting with some $\theta$ with respect to applied magnetic field. The average canting angle $\theta$ (the angle between the radiation direction and the magnetic moment direction) can be estimated from the area ratio of lines pair $(2,5)$ and $(1,6)$ by the relation [1]:

$$
\mathrm{A}_{2,5} / \mathrm{A}_{1,6}=4\left(1-<\cos ^{2} \theta>\right) /\left(3+3<\cos ^{2}>\right)
$$

where $A_{2,5}$ and $A_{1,6}$ are the areas of lines 2 and 5 and lines 1 and 6 , respectively. Using this relation, the average canting angle is calculated to be $32.3^{\circ}, 33.8^{\circ}$ and $48^{\circ}$ for samples $\mathrm{CO} 3, \mathrm{CO} 2$ and $\mathrm{CO} 1$, respectively. The small difference, observed in the cantin angle for samples $\mathrm{CO} 3$ and $\mathrm{CO} 2$ (coated and uncoated samples with the same particle size), is within the experimental error. 
In similar studies, spin canting was observed for coated particles of $\mathrm{CoFe}_{2} \mathrm{O}_{4}$ system, but this canting disappeared after removing the oleic acid [7]. However, the oleic acid was removed by heating the samples at $600^{\circ} \mathrm{C}$. During the heating of the sample at $600^{\circ} \mathrm{C}$, one may expect an increase in the particle size. Thus the disappearance of the canting effect in that case may be mainly due to an increase in particle size rather than due to the removal of the oleic acid from the sample as suggested by Berkowitz [7]. Thus one may conclude that the observed canting is not be due to coating the surface with oleic acid. On the other hand the significant difference in the canting angle between the two samples $\mathrm{CO} 1$ and $\mathrm{CO} 3$ (both coated with oleic acid) could be attributed mainly to the difference of the particle size. The particle size is $10.3 \mathrm{~nm}$ for both $\mathrm{CO} 3$ and $\mathrm{CO} 2$ while for sample $\mathrm{CO} 1$ it is $4 \mathrm{~nm}$ (in diameter), as calculated from $\mathrm{X}$-ray diffraction patterns. The decrease in the area $\mathrm{A}_{2,5}$ when the particle size increases suggests that the non-collinear structure is located in the surface region.

Assuming that all the canted spins are located in the surface, one could estimate the thickness $r$ of the canted layer by using the relation [23]:

$$
A_{c}=\left(V_{t}-V_{c}\right) / V_{t}
$$

where $V_{t}$ and $V_{c}$ are the total and core volume of the particle, respectively. If the particles are spherical then one could use the relation [23]:

$$
A_{c}=\left[R^{3}-(R-r)^{3}\right] / R^{3}
$$

where $R$ is the particle radius and $A_{c}$ is the relative area of the canted component.

The values of $\mathrm{r}$, calculated by this equation were found to be $0.89,0.96$ and $0.87 \mathrm{~nm}$, for samples $\mathrm{CO} 3, \mathrm{CO} 2$ and $\mathrm{CO} 1$, respectively. The values of $\mathrm{r}$ are within experimental errors are the same. If the spin canting that observed for the cobalt-ferrite is due to a large magnetic anisotropy one expect a smaller value of $r$ for sample CO1.

Thus this result could be explained by a model of a ferrimagnetic core with a $0.91 \pm 0.05 \mathrm{~nm}$ thick canted surface layer. The atoms in core, therefore these spins are not perfectly aligned along the direction of the applied magnetic field, but make a canting angle [2]. For the ferrimagnetic core the atom spins will be collinear with the direction of the applied magnetic field.

At room temperature the spectra showed a magnetic splitting in addition to quadrupole doublet. The doublet disappears at low temperature, indicating that the presence of superparamagnetic nano particles. 
Finally one could conclude that the main effect of the oleic acid coating is increasing the doublet area at room temperature, due to the number of the particles that exhibit fast relaxation. Similar observation have been observed for feroxyhite particles coated with oleic acid, in which this behaviour was explained by a decrease in the particle (dipole-dipole) interaction due to variation in the distance between the particles.

\section{Conclusions:}

The canting effect observed is mainly due to the spin canted surface layer (surface effect). There is no indication of a significant influence due to coating the surface with oleic acid on the spin-canting. Coating the surface of the particles with oleic acid caused a decrease of the particles interaction. It is possible to control the growth of the ultrafine cobalt ferrite particles by adding oleic acid prior to heating.

\section{Acknowledgment}

This work has been done at Physics Department, Building 307, Technical uUniversity of Denmark, DK-2800 Lyngby, Denmark, So. Professor Steem Mørup is acknowledged for providing the measurement facilities.

\section{References:}

1. S. Mørup. J.A. Dumesic and H. Topsoe, in: Application of Mössbauer Spectrscopy, Vol. II, ed. R.L. Cohen (Academic Press, New York, 1980) p.1.

2. A. H. Morrish and K. Haenda, J. Magn. Magn. Mater. 35, 105 (1983).

3. J. M. D. Coey, Can. J. Phys. 65, 1210 (1987).

4. E. Murad and J.H. Johnston, "Mössbauer Spectroscopy Applied to Inorganic Chemistry", Vol. 2, ed. G.J. Long (plenum Press, New York, 1987) pp. 507-582.

5. A. Ochi, K. Watanabe, M. Kiyama, T. Shinjo, Y. Bando and T. Takada, J. Phys. Soc. Japan 50, 2777 (1981).

6. J. G. Na, D.H. Han, J.G. Zaho, and H.L. Luo, J. Appl. Phys. 76, 6900 (1994).

7. A. E. Berkowitz, J. A. Lahut and C.E. Vanburen, IEEE Trans. Magn. MAG-16, 184 (1980).

8. H. R. Rechnberg and F.A. Tourinho, Hyper. Inter. 67, 627 (1991).

9. S. Gangopadhyay, Y. Yang, G. C. hahjipanayis, V. papaefthymiu, C. M. Sornsen, and K.J. Klabunde, J. Appl. Phys. 76, 6319 (1994). 
10. D. H. Han, J.P. Wang, Y. B. Feng and H. L. Luo, J. Appl. Phys. 76, 6591 (1994).

11. K. J. Davies, S. Wells and S.W. Charles, J. Magn. Magn. Mater. 122, 24 (1993).

12. M. A. G. Soler, S. W. da Silva, V. K. Garg, A. C. Olivera, R. B. Azevedo, A. C. M. Pimenta, E. C. D. Lima and P. C. Morais, Surface scie. 575, 12 (2005).

13. G. B. Ji, S. L.Tang, S. K.Ren, F. M. Zhang, B. X. Gu, and Y. W. Du, J. Crys. Grow. 270, 156 (2004).

14. L. D. Tung, V. Kolesnichenko, D. Caruntu, N. H. Chou, C. J. O'Connor, and L. Spinu, J. Appl. Phys. 93, 7486 (2003).

15. S. R. Ahmed, S. B. Ogale and P. Kofins, IEEE Trans. On Magne. 39, 2198 (2003).

16. M. Rajendran, R.C. Pullar, A.K. Bhattacharya, D. Das, S.N. Chintalapudi, C.K. Majumdar, J. Magn. Magn. Mat. 232, 71 (2001).

17. A. Slawska-Waniewska, P. Didukh, J.M.Greneche, P.C Fannin, J. Magn. Magn. Mat. 215, 227 (2000).

18. V. Masheva, M. Grigorova, N. Valkov, H.J. Blythe, T. Midlarz, V. Blaskov, J. Geshev and M. Mikhov, J. Magn. Magn. Mat. 196, 128 (1999).

19. Y. Ahn, E. Jung Choi, S. Kim and H. Nam Ok, Mater. Lett. 50, 47 (2001).

20. K. J. Davies, S. Wells, R. V. Upadhyay, S. W.Charles, K. O' Grady, M. El Hilo, T. M. M eaz and S. Mørup, J. Magn. Magn. Mat. 149, 14 (1995).

21. G. Bate, "Ferromagnetic materials", Vol. 2, p. 431, Ed. Wohlfrath (1980).

22. G.M. da Costa, E. De Grave, A.M. Bryan and L.H. Bowen, Hyper. Inter. 94, 1983 (1993).

23. T. M. Meaz, "Application of Mössbauer Spectrscopy to the study of small Magnetic Particles", Ph. D. Thesis, Tanta University (1995) 